Pacific Journal of Mathematics

SUBSPACES OF POSITIVE DEFINITE INNER PRODUCT 


\title{
SUBSPACES OF POSITIVE DEFINITE INNER PRODUCT SPACES OF COUNTABLE DIMENSION
}

\author{
WERNER BÄNI
}

We deal with the following problem, proposed by $I$. Kaplansky in 1950: If $V, \bar{V}$ are subspaces of an inner product space $(E, \Phi)$ of countable dimension over any field $k$, when does there exist a metric automorphism of $(E, \Phi)$ mapping $V$ onto $\bar{V}$ ? The present paper treats the case of positive definite symmetric spaces over $k=\boldsymbol{R}$. We shall characterize the orbits (under the orthogonal group of $(E, \Phi)$ ) of a large class of subspaces $V$ by two sequences of cardinals attached to $V$ in a natural way (if e.g., $V^{\perp}=0$ or $V=V^{\perp \perp}$ only a few of them are $\neq 0$; the case $V^{\perp}=0$ is covered by work of H. Gross). However, classifying the subspaces not in this class is equivalent to classifying vector spaces $F$ endowed with a sequence $\Omega_{0}, \Omega_{1}, \Omega_{2}, \cdots$ of positive definite forms.

\section{Introduction.}

1.1. Let $E$ be a real vector space of countable dimension, equipped with a positive definite symmetric bilinear form $\Phi: E \times E \rightarrow$ $\boldsymbol{R}$. We are concerned with the problem of classifying subspaces $V$ of $E$ with respect to metric automorphisms of $(E, \Phi)$ or, in other words, of describing the orbits of subspaces of $E$ under the action of the orthogonal group of $(E, \Phi)$.

1.2. This problem of course originates from the wish to know how Witt's theorem generalizes to spaces of infinite dimension (the celebrated theorem says that every isometry between subspaces of a finite dimensional inner product space can be extended to the whole space). In fact, Kaplansky ([6], question 3, p. 16) stated the problem explicitly for arbitrary inner product spaces of countable dimension over any field $k$ (in the case of uncountable dimensions the problem seems too nasty). Confirming a conjecture of Kaplansky, Gross [2] showed that in the presence of "sufficiently many" isotropic vectors (e.g., if $\Phi$ is alternate or if $k$ is quadratically closed with char $(k) \neq 2$ and $\Phi$ is symmetric) the orbit of a subspace $V$ may be characterized by seven cardinal numbers, namely the codimensions of neighboring spaces in the sublattice $\mathfrak{J}$ of $\mathfrak{L}(E)$ (the lattice of all linear subspaces of $E$ ) generated $\perp$-stably by $V$ (i.e., $X \in \mathfrak{\Im} \Longrightarrow X^{\perp} \in \Im$ $)$ ).

1.3. For positive definite forms over ordered fields - a case at the other extreme - the problem seems to be considerably harder. 
For nice enough subfields $k$ of $\boldsymbol{R}$ Gross [3], [5] and Schneider [8] have characterized the orbit of $V$ by (at most) four matrices of (possibly) infinite size over $\boldsymbol{R}$, subject to a complicated equivalence relation. It will follow from our interpretation that in fact, in the case of a completely general $V$, they do not simplify the classification problem, even if $k=\boldsymbol{R}$ (see 2.8 below). For $\perp$-dense subspaces $V$ (i.e., $V^{\perp}=0$ ), however, their result permits a satisfactory insight. In the dense case the problem amounts to the task of classifying positive semidefinite symmetric $k$-bilinear maps $\Psi: F \times F \rightarrow \boldsymbol{R}$ on $k$-spaces $F$ of countable dimension. If $k=\boldsymbol{R}, \Psi$ is an inner product on $F$, characterized by the dimension of its radical $r \Psi=F^{\perp}$ and of $F / r \Psi$. But if e.g., $k=\boldsymbol{Q}$ the problem is roughly the same as that of classifying sequences $\Psi_{0}, \Psi_{1}, \Psi_{2}, \cdots$ of symmetric forms on $F$ (since $\boldsymbol{R} \cong \boldsymbol{Q}^{\boldsymbol{\aleph}_{0}}$ as a vector space over $\boldsymbol{Q}$ ). This suggests that a reasonable classification for arbitrary subspaces $V$ can be expected at most in the case $k=\boldsymbol{R}$, whence the assumptions put down in 1.1 (which will be in force from now on). Indeed, it will be possible to describe the orbits of a large class of subspaces, but even here we shall be left with an "unsolvable" problem (2.6).
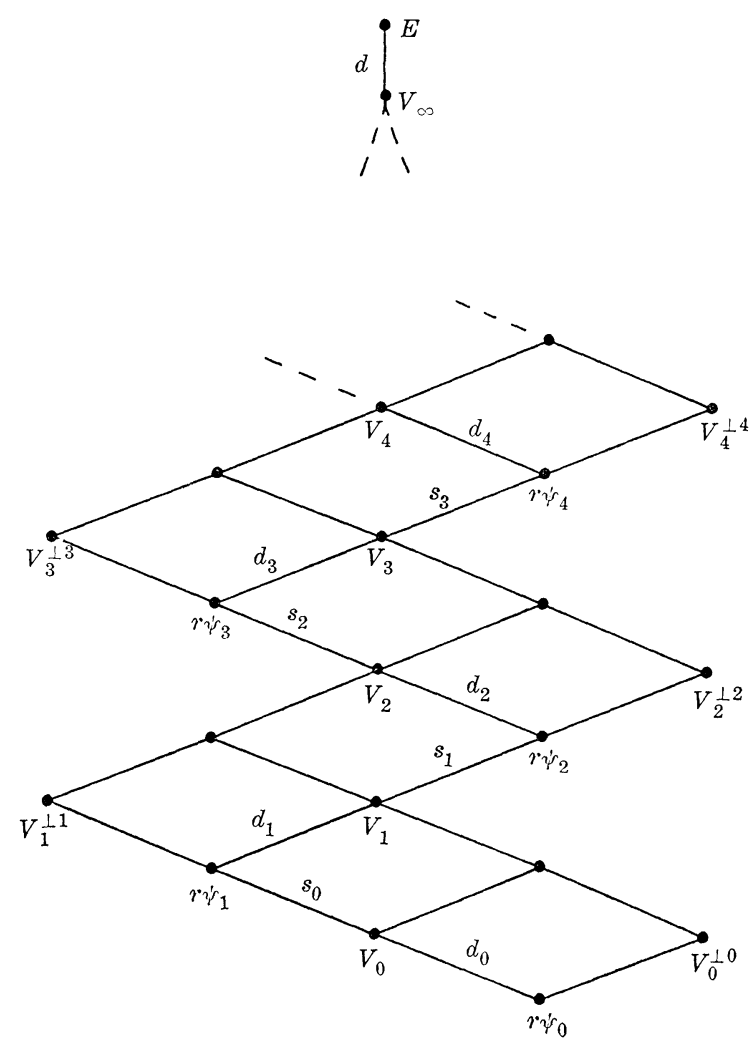

FiguRe 1 

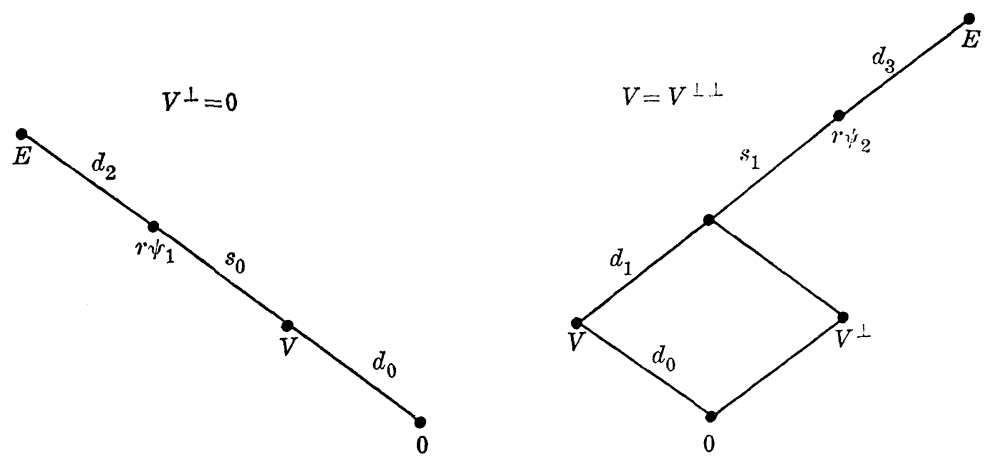

FIGURE 2

1.4. In this paper we shall associate to $(E, \Phi, V)$, in a certain natural way, a lattice $\mathfrak{v}$ consisting of subspaces of $E$, as depicted above (Fig. 1; the definitions are given in 2.3). If $V_{\infty}=E$ (which is always the case if $\mathfrak{b}$ is finite) the codimensions $d_{i}, s_{i}$ in Fig. 1 form a complete set of invariants of the orbit of $V$. If e.g., $V$ is $\perp$ dense or $\perp$-closed $\left(V^{\perp \perp}=V\right)$ the lattices are finite and look as in Fig. 2. If, however, $V_{\infty} \neq E$ an additional invariant of a different kind appears: A vector space $F\left(\cong E / V_{\infty}\right)$, endowed with a sequence $\left(\Omega_{i}\right)_{i \in N}$ of positive definite forms $\Omega_{i}$ such that for each nonzero $x \in F$ one has $\Omega_{0}(x, x)>\Omega_{1}(x, x)>\Omega_{2}(x, x)>\cdots$. Two such objects $\left(F,\left(\Omega_{i}\right)\right)$, $\left(F^{\prime \prime},\left(\Omega_{i}^{\prime}\right)\right)$ are equivalent if there is an isomorphism $S: F \rightarrow F^{\prime \prime}$ such that for all $x, y \in F$ we have $\Omega_{i}^{\prime}(S x, S y)=\Omega_{i}(x, y)$ for almost all $i \in N$. In 2.6 we shall give some indications why a classification of such objects is out of reach.

Chapter 3 contains our main result: The codimensions $d_{i}, s_{i}$ in the lattice $\mathfrak{b}$ together with $\left(F,\left(\Omega_{i}\right)\right)$ characterize the orbit of $V$. In Chapter 4 we investigate how the different invariants can be combined, and as an application we show in Chapter 5 how $(E, \Phi, V)$ can be decomposed orthogonally into components of simpler structure.

1.5. Occasionally we make use of the possibility of embedding the space $(E, \Phi)$ into a Hilbert space. Nevertheless our problem is a purely algebraic one; ther is no overlapping withHilbert space theory.

One final remark: All the results of this paper are expressed for symmetric forms over $\boldsymbol{R}$, but it should be clear that they hold mutatis mutandis also for hermitean forms over $\boldsymbol{C}$ or $\boldsymbol{H}$ (endowed with their usual involutions).

2. Definition of the invariants.

2.1. A triple $\mathfrak{C}=(E, \Phi, V)$, where $(E, \Phi)$ is a symmetric, positive definite inner product space of countable dimension over $R, V$ a 
subspace of $E$, will be called an embedding. Two embeddings are congruent, $\widetilde{C} \cong \mathbb{C}^{\prime}$, if there is an isometry $T:(E, \Phi) \rightarrow\left(E^{\prime}, \Phi^{\prime}\right)$ with $T V=V^{\prime}$. Countable orthogonal direct sums of embeddings are formed in the obvious way.

2.2. We may embed $(E, \Phi)$ canonically into a Hilbert space $(\widetilde{E}, \widetilde{\Phi})$ : Let $\widetilde{E}$ be the completion of $E$ with respect to the norm induced on $E$ by $\Phi$, and $\widetilde{D}$ the natural extension of $\Phi$ to $\widetilde{E} \times \widetilde{E}$. For an arbitrary subspace $W \subset E$ we have a decomposition $\widetilde{E}=\widetilde{W} \oplus \widetilde{W}^{\lrcorner}$where $\widetilde{W}$ is the norm closure of $W$ in $\widetilde{E}$. Define a new positive form $\widetilde{T}$ on $\widetilde{E}$ by $\widetilde{\Psi}(x, y)$ : $=\widetilde{\Phi}\left(x^{\prime}, y^{\prime}\right)$ with $x^{\prime}, y^{\prime}$ the projections of $x, y$ in $\widetilde{W}^{\lrcorner}$. We are interested in its restriction $\Psi=\left.\widetilde{\Psi}\right|_{E \times E}$ which may be described as follows. Take any orthonormal basis ${ }^{1}\left(w_{i}\right)$ of $W$; then for $x, y \in E$ we have

$$
\Psi(x, y)=\Phi(x, y)-\sum_{i} \Phi\left(x, w_{\imath}\right) \Phi\left(w_{i}, y\right)
$$

We shall use the suggestive notation $\Psi=\Phi-\Phi W \Phi$. The radical $r \Psi=\{x \in E \mid \Psi(x, y)=0 \forall y \in E\}=\widetilde{W} \cap E$ coincides with the norm closure of $W$ in $E$. If $\Phi$ is merely positive instead of positive definite we consider the Hilbert space belonging to $E / \imath \Phi$ in order to get our $\Psi$. Again (1) is valid if $\left(w_{i}\right)$ is an orthonormal basis of a complement of $r \Phi \cap W=\left.r \Phi\right|_{W \times W}$ in $W$. As a direct consequence of the definitions (but not of (1)!) we get

LEMma. Let $W_{1} \subset W_{2} \subset E$ and $\Psi=\Phi-\Phi W_{1} \Phi$. Then $\Psi-\Psi W_{2} \Psi=$ $\Phi-\Phi W_{2} \Phi$.

2.3. We shall now construct the lattice $\mathfrak{v}=\mathfrak{b}(\mathfrak{5})$ pictured in Fig. 1. For any embedding $\mathfrak{F}=(E, \Phi, V)$ we define by recursion a sequence $\left(V_{2}\right)_{N}$ of subspaces of $E$ and a sequence $\left(\Psi_{i}\right)_{N}$ of positive forms on $E$, as follows.

$$
\begin{aligned}
& \Psi_{0}:=\Phi, V_{0}:=V \\
& \Psi_{\imath+1}:=\Psi_{i}-\Psi_{i} V_{i} \Psi_{i}, V_{i+1}:=r \Psi_{i+1}+V_{i}^{\perp i} .
\end{aligned}
$$

Here, for any subspace $X \subset E$, let $X^{\iota_{\imath}}=\left\{y \in E \mid \forall x \in X: \Psi_{i}(x, y)=0\right\}$. Note that $\Psi_{i+1}=\Phi-\Phi V_{i} \Phi$ by Lemma 2.2. In the proof of the congruence Theorem 3.1, however, we shall need the recursive description (2). Now let $\mathfrak{b}$ be the smallest complete sublattice of the lattice $2(E)$ of all subspaces of $E$ which contains $0, E, V$ and which is $\perp_{\imath}$-stable for all $i \in N$ (i.e., $X \in \mathfrak{b} \Rightarrow X^{\llcorner\imath} \in \mathfrak{b}$ ). It is readily verified that indeed $\mathfrak{b}$ is given by the diagram in Fig. 1, with $V_{\infty}=\bigcup_{i \in N} V_{i}$

1 A basis in the algebraic sense, i.e., a so called Hamel basis. The word "basis" will always be used in this sense. 
(as to $\perp_{i}$-stability, use the lemma below). We shall see in Chapter 4 that in the most general case all the spaces in Fig. 1 are different from each other. For later reference we note

Lemma. For $x \in V_{\imath}^{\lrcorner, i}, y \in E$ one has $\Psi_{i \uparrow 1}(x, y)=\Psi_{\imath}(x, y)$. Hence $V_{i+1}^{j i+1}=V_{i}^{\perp i_{i}}$.

Proof. Follows from (1) and (2).

2.4. The indices (codimensions of neighboring spaces) in the lattice $\mathfrak{b}$ are the following ones

$$
\left.\begin{array}{l}
d_{\imath}:=\operatorname{dim} V_{\imath} / r \Psi_{\imath} \\
s_{\imath}:=\operatorname{dim} r \Psi_{\imath+1} / V_{i} \\
d:=\operatorname{dim} E / V_{\infty}
\end{array}\right\} \quad i \geqq 0 .
$$

We also have $d_{i+1}=\operatorname{dim} V_{i}^{\iota_{2}} / v \cdot \Psi_{\imath}$. The question of independence will be settled in Chapter 4, but we already note the following

Lemma. If $d_{i}<\boldsymbol{W}_{0}$ for some $i$ then $E=V_{i}+V_{i-2}, i_{\circ} e_{.}, d_{j}=0$ for $j \geqq i+2$ and $d=0$. Such an $i$ exists if and only if $\mathfrak{v}$ is finite.

Proof. Clearly, since the finite dimensional image of $V_{i}$ in $E / r \Psi_{i}$ must be an orthogonal summand in the positive definite space $\left(E, \Psi_{i}\right) / r \Psi_{i}$.

2.5. Suppose that $d \neq 0$ and set $E=V_{\infty} \oplus F$. Let $\Omega_{\imath}$ be the restriction of $\Psi_{2}$ to $F \times F$. For every nonzero $x \in F$ and for all $i \in N$ we have

$$
\Omega_{\imath}(x, x)>\Omega_{i+1}(x, x)>0
$$

since by (1), $\Omega_{i}(x, x)=\Omega_{i+1}(x, x)$ would mean that $x \in V_{i}^{\llcorner} \subset V_{\imath+1} \subset V_{\infty}$. If $F^{\prime}$ is another complement of $V_{\infty}$ in $E$ or, more generally, (5' is an embedding congruent to $\subseteq$ by $T: E \widetilde{C} E^{\prime}=V_{\infty}^{\prime} \oplus F^{\prime}$ then, since $T V_{\infty}=V_{\infty}^{\prime}, T$ induces an isomorphism $S: F \rightarrow F^{\prime \prime}$ with

(5) to $x, y \in F$ there exists $i \in N$ such that $\Omega_{j}^{\prime}\left(S_{x}, S y\right)=\Omega_{j}(x, y)$ for all $j \geqq i$.

Of course, if $d=\operatorname{dim} F<\infty$ we may replace (5) by $\left(5^{\prime}\right)$ for almost all $i \in N, S$ is an isometry $\left(F, \Omega_{i}\right) \rightarrow\left(F^{\prime}, \Omega_{i}^{\prime}\right)$. Let us call the object $\left(F,\left(\Omega_{i}\right)\right.$ ) (determined by $\sqrt{5}$ up to an isomorphism with $(5)$ ) the top of the embedding $\left(5\right.$. Two tops $\left(F,\left(\Omega_{2}\right)\right),\left(F^{\prime \prime},\left(\Omega_{i}^{\prime}\right)\right)$ are said to be isomorphic if there is an isomorphism $S: F \rightarrow F^{\prime}$ which satisfies (5). 
We shall see in 4.2 that the only restrictions on the top are $\operatorname{dim} F \leqq \aleph_{0}$ and (4).

2.6 We are going to make some comment on the "wildness" of the classification problem of tops as defined in 2.5. Since we do not want to go into the details they have to remain somewhat vague. Let $n$ be a fixed natural number and consider finite dimensional vector spaces $F$ together with $n$ symmetric forms $\Gamma_{1}, \cdots, \Gamma_{n}$ on $F$, to be classified with respect to simultaneous isometry. We start from the "well known" fact that this is a "wild" problem" for $n \geqq 3$. It is plausible that it will make no essential difference if we require the $\Gamma_{i}$ to be positive definite. But then we can make $\left(F ; \Gamma_{1}, \cdots, \Gamma_{n}\right)$ into a top in the following way: Let $\lambda$ be the largest positive real number with $\lambda\left(\Gamma_{1}+\cdots+\Gamma_{n}\right)(x, x) \leqq \Gamma_{n}(x, x)$ (all $\left.x \in F\right)$ and put $\Omega_{0}=\Gamma_{1}+\cdots+\Gamma_{n}, \Omega_{1}=\Gamma_{2}+\cdots+\Gamma_{n}, \cdots, \Omega_{n-1}=\Gamma_{n}, \Omega_{n}=$ $(\lambda / 2) \Omega_{0}, \Omega_{i}=(\lambda / 2) \Omega_{i-n}$ for $i \geqq n$. Since (4) is now satisfied $\left(F,\left(\Omega_{i}\right)\right)$ is a top. Using $\left(5^{\prime}\right)$ it is easy to see that the assignment $\left(F ; \Gamma_{1}, \cdots, \Gamma_{n}\right) \mapsto$ $\left(F,\left(\Omega_{i}\right)\right)$ induces an injection from the set of isomorphism classes of objects $\left(F ; \Gamma_{1}, \cdots, \Gamma_{n}\right)$ into the set of isomorphism classes of tops.

2.7. Let $(5)$ be any embedding and put $(\bar{E}, \bar{\Phi}):=\left(E, \Psi_{1}\right) / r \Psi_{1}$. Let $\bar{V}$ be the image of $V^{\perp}$ (or of $V_{1}$ ) in $\bar{E}$ and consider the embedding $\bar{F}$ : = $(\bar{E}, \bar{\Phi}, \bar{V})$.If $\bar{V}_{i}, \bar{\Psi}_{i}$ are the objects associated to it by $(2)$ we see that $\left(\bar{E}, \bar{\Psi}_{i}\right)=\left(E, \Psi_{i+1}\right) / r \Psi_{1}$ and that $\bar{V}_{i}$ coincides with the image of $V_{i+1}$ in $\bar{E}$. Hence $\bar{d}_{i}=d_{i+1}$ and $\bar{s}_{i}=s_{i+1}$. The top of $\overline{\mathbb{C}}$ is derived from the top of $C$ by dropping $\Omega_{0}$, i.e., $\bar{\Omega}_{i}=\Omega_{i+1}$.

2.8. It will follow from our congruence Theorem 3.1 that $\sqrt{ } \cong \mathbb{E}$ if and only if $\overline{\mathbb{C}}=\overline{\mathbb{C}}^{\prime}$ and $d_{0}=d_{0}^{\prime}, s_{0}=s_{0}^{\prime}$. If one introduces standard bases in the sense of [8] in order to express this result in matrix terms one gets exactly the congruence theorem of Schneider's [8] (Satz IV. 3). By 2.7 this result is virtually useless in the general case. On the other hand, for embeddings with finite lattice $\mathfrak{v}$ the congruence Theorem 3.1 can be recovered from it by iterated application.

\section{The congruence theorem.}

\subsection{With the notations of 2.4 and 2.5 we have:}

2 The term "wild" or "unclassifyable" is to be interpreted in the spirit of [1], p. 444-06/07. Indeed, from Example 4.3 in [7] it follows that the problem of classifying the objects $\left(F ; \Gamma_{1}, \cdots, \Gamma_{n}\right)$ is equivalent to the problem of classifying the representations of the quiver $\stackrel{\longrightarrow}{\longrightarrow}$ ( $n$ arrows). 
THEOREM. Two embeddings $(E, \Phi, V),\left(E^{\prime}, \Phi^{\prime}, V^{\prime}\right)$ are congruent if and only if $d_{i}=d_{i}^{\prime}, s_{i}=s_{i}^{\prime}$ for all $i \in N$, and their tops are isomorphic.

The rest of this chapter is devoted to the proof of the nontrivial half of this theorem. We shall construct an isomorphism $T: E \rightarrow E^{\prime}$ which is an isometry $\left(E, \Psi_{i}\right) \rightarrow\left(E^{\prime}, \Psi_{i}^{\prime}\right)$ for all $i \in N$, and with $T A=$ $A^{\prime}$ for every $A \in \mathfrak{v}$. Here $A^{\prime}$ is the lattice element of $\mathfrak{v}^{\prime}$ corresponding to $A$; we shall use this notation throughout. For the construction of $T$ we use a method developed by Gross in [4] combined with a kind of induction which is made possible by 2.7. Those verifications which can be taken over from [4] will be suppressed.

3.2. The induction just mentioned will be based on the case of $\perp$-dense embeddings of finite codimension, which is already done in [3]. We shall not repeat the proof of this but state as a lemma what we need.

Lemma. Let $\sqrt{5}, \mathfrak{C}^{\prime}$ be $\perp$-dense embeddings with $\operatorname{dim} E / V=$ $\operatorname{dim} E^{\prime} / V^{\prime}<\infty$. Suppose we are given an isomorphism $T: X \rightarrow X^{\prime}$ between finite dimensional subspaces $X, X^{\prime}$ of $E, E^{\prime}$ such that $E=$ $V+X, E^{\prime}=V^{\prime}+X^{\prime}, T(X \cap V)=X^{\prime} \cap V^{\prime}$, and such that $T$ is an isometry for each of the restrictions of $\Phi, \Phi^{\prime}$ and $\Psi_{1}, \Psi_{1}^{\prime}$. If $v \in V$, $v \notin X$ there exists $v^{\prime} \in V^{\prime}, v^{\prime} \notin X^{\prime}$ with $\Phi^{\prime}\left(v^{\prime}, T x\right)=\Phi(v, x)($ all $x \in X)$ and $\Phi^{\prime}\left(v^{\prime}, v^{\prime}\right)=\Phi(v, v)$.

Proof. This can be extracted from [3], I.2 and III.1 or from [8], III.9.

3.3. The construction of an isomorphism $T$ as indicated in 3.1 is accomplished by a simple recursion (as e.g., in [4]) if we are able to show that the following construction problem (6) can always be solved. Suppose that $\sqrt{ } \sqrt{ }, \mathfrak{C}^{\prime}$ satisfy the assumptions of the theorem, and let the isomorphism $S: F \rightarrow F^{\prime}$ satisfy (5), where $E=V_{\infty} \oplus F$, $E^{\prime}=V_{\infty}^{\prime} \oplus F^{\prime \prime}$.

(6) Let $T: X \rightarrow X^{\prime}$ be an isomorphism between finite dimensional subspaces $X, X^{\prime}$ of $E, E^{\prime}$ such that the following hold

( $\alpha) \quad(X+A) \cap(X+B)=X+(A \cap B)$ for every $A \in \mathfrak{v}$;

$(\alpha)^{\prime}$ the corresponding property for $X^{\prime}$ with respect to $\mathfrak{b}^{\prime}$;

( $\beta) ~ T(X \cap A)=X^{\prime} \cap A^{\prime}$ for every $A \in \mathfrak{b}$;

( $\gamma$ ) for each $i \in N, T$ is an isometry for the restrictions of $\Psi_{i}, \Psi_{i}^{\prime}$;

$(\delta)$ if $Z, Z^{\prime}$ are the projections of $X, X^{\prime}$ in $F, F^{\prime \prime}$ respectively the isomorphism $Z \rightarrow Z^{\prime}$ induced by $T$ (in view of $(\beta)$ ) coincides with the restriction of $S$.

If $y \in E, y \notin X$ there exist finite dimensional subspaces $X_{1}, X_{1}^{\prime}$ of $E ; E^{\prime}$ such that $X+R y \subset X_{1}, X^{\prime} \subset X_{1}^{\prime}$ and an isomorphism $T_{1}: X_{1} \rightarrow X_{1}^{\prime}$ 
extending $T$ such that $(\alpha)$ through $(\delta)$ hold for $X_{1}, X_{1}^{\prime}, T_{1}$ in lieu of $X, X^{\prime}, T$.

3.4. In view of $(\alpha)$ the set $\{A \in \mathfrak{v} \mid y \in X+A\}$ is a sublattice of $\mathfrak{v}$, and since $\mathfrak{v}$ satisfies the minimum condition it has a smallest element $D=D(y, X)$. We may assume that $y \in D$. The minimum condition also allows to assume (by induction) that the construction problem can be solved in all situations with a $D$ properly smaller than the present one. In particular, we may assume that $D$ is not the sum of two smaller elements of $\mathfrak{v}$ (i.e., $D$ is sup-irreducible), otherwise, by applying the induction assumption twice, we are done. So $D$ has an immediate predecessor $D_{0} \subsetneq D$ in $\mathfrak{v}$. The same reasoning shows that we may allow ourselves to replace $y$ by a vector $y_{1}=$ $y+v$ with $v \in D_{0}$, if necessary. We still have

$$
y_{1} \in D, y_{1} \notin X+D_{0} \text {. }
$$

We shall set $X_{1}=X \oplus \boldsymbol{R} y_{1}, X_{1}^{\prime}=X^{\prime} \oplus \boldsymbol{R} y_{1}^{\prime}, T_{1} Y_{1}=y_{1}^{\prime}$ for some $y_{1}^{\prime} \in E^{\prime}$ with

$$
y_{1}^{\prime} \in D^{\prime}, \quad y_{1}^{\prime} \notin X^{\prime}+D_{0}^{\prime} .
$$

Recall that for $A \in \mathfrak{v}, A^{\prime}$ denotes the corresponding lattice element of $\mathfrak{v}^{\prime}$. By $\left(7^{\prime}\right)$ we have $D^{\prime}=D\left(y_{1}^{\prime}, X^{\prime}\right)$. The conditions $(\alpha),\left(\alpha^{\prime}\right),(\beta)$ are then verified exactly in the same way as in [4]; hence we shall concentrate upon $(\gamma)$ and $(\delta)$. We shall distinguish the following cases: (a) $D=V$, (b) $D=V^{\perp}$, (c) $D=r \Psi_{1}$, (d) $r \Psi_{1} \varsubsetneqq D \subset V_{\infty}$, (e) $D=E \neq V_{\infty}$. Notice that condition ( $\delta$ ) needs attention only in case (e).

3.5. The case $D=V$. In order to save $(\gamma)$ we only have to find $y^{\prime} \in V^{\prime}, y^{\prime} \notin X^{\prime}$ such that

$$
\Phi^{\prime}\left(y^{\prime}, T x\right)=\Phi(y, T x)(\text { all } x \in X), \quad \Phi^{\prime}\left(y^{\prime}, y^{\prime}\right)=\Phi(y, y)
$$

since $V \subset r \Psi_{i}$ for $i \geqq 1$. Set $X=X_{0} \oplus^{\perp}$ with $X_{0}=X \cap V^{\perp}$ and similarly for $X^{\prime}$. By $(\beta)$ and $(\gamma)$ we have $T X_{0}=X_{0}^{\prime}, T U=U^{\prime}$. Now it suffices to require (8) for $x \in U$. Consider the embeddings $V \subset E_{0}=$ $V+U, V^{\prime} \subset E_{0}^{\prime}=V^{\prime}+U^{\prime}$. Since $V^{\lrcorner} \cap E_{0}=V^{\perp} \cap(V+U) \subset\left(X+V^{\perp}\right) \cap$ $(X+V)=X$ by $(\alpha)$, we have $V^{\perp} \cap E_{0}=V^{\perp} \cap X \cap E_{0}=0$ and similarly $V^{\perp} \cap E_{0}^{\prime}=0$. Thus we may cite Lemma 3.2 to get what we want.

3.6. The case $D=V^{\perp}$. Here we shall use 2.7, i.e., we consider our problem modulo $r \Psi_{1}, r \Psi_{1}^{\prime}$. It is easy to verify that $(\alpha)$ through $(\delta)$ remain valid in $\bar{\complement}, \overline{\widetilde{C}}^{\prime}$. Since $y \notin X+r \Psi_{1}$ (otherwise $y \in X$ by $(\alpha)$ ) we may apply 3.5 to $\overline{\mathfrak{C}}, \overline{\mathbb{C}^{\prime}}$ in order to find $y^{\prime} \in V^{\prime \perp}, y^{\prime} \notin X^{\prime}$ such that for $i \geqq 1$ one has 


$$
\Psi_{i}^{\prime}\left(y^{\prime}, T x\right)=\Psi_{i}(y, x)(x \in X), \quad \Psi_{i}^{\prime}\left(y^{\prime}, y^{\prime}\right)=\Psi_{i}(y, y) .
$$

But, by the lemma in 2.3, (9) is valid for $i=0$ as well, whence $(\gamma)$ holds for $X_{1}, X_{1}^{\prime}, T_{1}$.

3.7. The case $D=r \Psi_{1} \supsetneq V$. Here $D_{0}=V$ and $\operatorname{dim} V=\boldsymbol{\aleph}_{0}$. We have to find $y_{1}^{\prime} \in r_{1}^{\prime}, y_{1}^{\prime} \notin X^{\prime}+V^{\prime}$ and $v \in V$ such that for $y_{1}=y+v$ we have

$$
\Phi^{\prime}\left(y_{1}^{\prime}, T x\right)=\Phi\left(y_{1}, x\right)(x \in U), \quad \Phi^{\prime}\left(y_{1}^{\prime}, y_{1}^{\prime}\right)=\Phi\left(y_{1}, y_{1}\right)
$$

where $U$ is defined as in 3.5. To this end, start with any $y^{\prime} \in r \Psi_{1}^{\prime}$, $y^{\prime} \notin X^{\prime}+V^{\prime}$. Such a choice is possible since $\operatorname{dim} r \Psi_{1} / r \Psi_{1} \cap(X+V)=$ $\operatorname{dim} r \Psi_{1}^{\prime} / r \Psi_{1}^{\prime} \cap\left(X^{\prime}+V^{\prime}\right)$ (this follows from $(\beta)$ and from the equation $\operatorname{dim} r \Psi_{1} / r \Psi_{1} \cap(X+V)=\operatorname{dim} r \Psi_{1} /\left(r \Psi_{1} \cap X\right)+V=\operatorname{dim} r \Psi_{1} / V-\operatorname{dim} X \cap$ $\left.r \Psi_{1} / X \cap V\right)$. Since $V^{\lrcorner} \cap U=0$ there is $v_{0} \in V$ such that $\Phi\left(y+v_{0}, x\right)=$ $\Phi^{\prime}\left(y^{\prime}, T x\right)$ for all $x \in U$ (this follows since $V+U^{\perp}=E$; see also Lemma 5 in [6]). Supsose that $\lambda=\Phi^{\prime}\left(y^{\prime}, y^{\prime}\right)-\Phi\left(y+v_{0}, y+v_{0}\right)>0$. Then we may choose $v_{1} \in V \cap\left(X+\boldsymbol{R}\left(y+v_{0}\right)\right)^{\perp}$ (a space of infinite dimension!) with $\Phi\left(v_{1}, v_{1}\right)=\lambda$ and set $y_{1}=y+v_{0}+v_{1}, y_{1}^{\prime}=y^{\prime}$. If $\lambda<0$ we may apply a similar correction to $y^{\prime}$ in order to adjust its length.

3.8. The case $r \Psi_{1} \varsubsetneqq D \subset V_{\infty}$. Again we reduce modulo $r \Psi_{1}, r \Psi_{1}^{\prime}$. The position of $D(\bar{y}, \bar{x})=\bar{D}$ (the image of $D$ in $\bar{E}=E / r \Psi_{1}$ ) in $\overline{\mathfrak{v}}=$ $\mathfrak{v}(\overline{\mathbb{C}})$ being lower than that of $D$ in $\mathfrak{v}$ we are able to apply in induction argument similar to that in 3.4 (the induction starts from 3.5, 3.6 and 3.7). This provides us with a vector $y^{\prime} \in D^{\prime}, y^{\prime} \notin X^{\prime}+D_{0}^{\prime}$ which fulfills (9) for $i \geqq 1$. If we modify $y$ to $y_{1}$ and $y^{\prime}$ to $y_{1}^{\prime}$ by adding vectors from $V \subset D_{0}, V^{\prime} \subset D_{0}^{\prime}$ we do not affect the validity of (9) for $i \geqq 1$. But the arguments in 3.7 show that such modifications also suffice to arrange for the validity of (9) for $i=0$.

3.9. The case $D=E \neq V_{\infty}$. We have $D_{0}=V_{\infty}, y \notin X+V_{\infty}$. Let $z$ be the projection of $y$ in $F$ (hence $z \notin Z$ ) and let $y^{\prime}=S z$. By $(\delta)$ we have $y^{\prime} \notin V_{\infty}^{\prime}+X^{\prime}$, hence $\left(7^{\prime}\right)$. From (5) and the choice of $y^{\prime}$ we infer that there exists $n \in N$ such that (9) is fulfilled for $i \geqq n$. It is now easy to modify $y$ and $y^{\prime}$ modulo $r \Psi_{n}$ and $r \Psi_{n}^{\prime}$ in such a way that (9) becomes valid for all $i \in N$. Indeed, an induction argument reduces the problem to the case $n=1$, where again we proceed as in 3.7. This settles condition $(\gamma)$ for $X_{1}, X_{1}^{\prime}, T_{1}$, and $(\delta)$ is clear by the choice of $y^{\prime}$.

The proof of the congruence theorem is thus complete.

4. Independence of the invariants. 
4.1. For the purpose of constructing embeddings with given top and given cardinal invariants $d_{i}, s_{i}$ the Hilbert space setting of 2.2 is an appropriate tool. We begin with a result concerning 2.7.

Proposition. If $\mathbb{F}^{\prime}$ is any embedding and $s \leqq \aleph_{0}$ there is an embedding $\sqrt{5}$ such that $\mathbb{F}^{\prime} \cong \overline{\mathfrak{F}}$ (notation of 2.7) and $s=\operatorname{dim} r \Psi_{1} / V$. If $V^{\prime}=E^{\prime}$ and $s=0$ then $\operatorname{dim} V$ may be chosen arbitrarily, otherwise $\operatorname{dim} \mathrm{V}=\boldsymbol{\aleph N}_{0}$.

Proof. Let $H_{1}=\widetilde{E}^{\prime}$ be the Hilbert space associated to $\left(E^{\prime}, \Phi^{\prime}\right)$ and let $H_{0}$ be any (infinite dimensional separable) Hilbert space. Choose subspaces $V \subset R \subset H_{0}$ with $\operatorname{dim} V=\boldsymbol{\aleph}_{0}, \operatorname{dim} R / V=s, V$ dense in $H_{0}$. Let $\widetilde{\Phi}$ be the form on $H=H_{0} \oplus H_{1}$ and $p: H \rightarrow H_{1}$ the orthogonal projection. We have to specify an $\boldsymbol{\aleph}_{0}$-dimensional dense subspace $E \subset H$ such that

$$
E \cap H_{0}=R, \quad p(E)=E^{\prime}, V^{\prime}=V^{\perp} \cap E=E \cap H_{1}
$$

holds. To this end, set $E^{\prime}=V^{\prime} \oplus X_{1}$, choose a subspace $X_{0} \subset H_{0}$ with $R \cap X_{0}=0$ together with an isometry $S: X_{0} \rightarrow X_{1}$, and put $E$ : = $R+\left\{x+S x \mid x \in X_{0}\right\}+V^{\prime}$. Now the verification of (11) and of the density of $E$ is easy. With $\Phi:=\widetilde{\Phi} \mid E \times E$ the embedding $\mathbb{F}=$ $(E, \Phi, V)$ certainly has the required property (with $r \Psi_{1}=R$ ). If $V^{\prime}=E^{\prime}$ and $s=0$ we do not need the above construction. In this case we may set $E=V \bigoplus^{\perp} E^{\prime}$ with arbitrary $\operatorname{dim} V \leqq \aleph_{0}$. But if $V^{\prime} \neq E^{\prime}$ Lemma 2.4 implies $\operatorname{dim} V=\boldsymbol{\aleph}_{0}$, and if $s \neq 0$ the same conclusion holds since $r \Psi_{1} \subset V^{\perp \perp}$ by Lemma 2.3 .

COROLlaRY 1. For embeddings $\sqrt{5}$ with finite lattice $\mathfrak{v}$ the only restrictions on the numbers $d_{i}, s_{i} \leqq \boldsymbol{\aleph}_{0}$ are the following. Let $n$ be the smallest natural number with $d_{n}<\infty$. Then

(a) $d_{i}=\aleph_{0}$ for $i<n$ and $d_{i}=0$ for $i>n+1$.

(b) $s_{i}=0$ for $i \geqq n$.

This follows from the proposition and from Lemma 2.4. By taking orthogonal sums of countable families of such embeddings we get

COROLlary 2. For embeddings (5) with infinite lattice $\mathfrak{v}$ but zero top the only restrictions on $d_{i}, s_{i}$ are: $d_{i}=\aleph_{0}$ for all $i \in N$.

4.2. If $\sqrt{ }$ is an embedding with nonzero top (2.5) then all $d_{i}=\aleph_{0}$. We now want to show that there is no connection between the top and the $s_{i}$.

Proposition. Let $F$ be a vectorspace of countable dimension 
endowed with a strictly decreasing sequence of positive definite symmetric forms $\Omega_{i}\left(i . e .\right.$, (4) holds). Then there is an embedding $\mathbb{C}^{\prime}=$ $\left(E^{\prime}, \Phi^{\prime}, V^{\prime}\right)$ with top $\left(F,\left(\Omega_{i}\right)\right)$ and prescribed values of the $s_{i}$.

Proof. Start with an embedding (5) which has infinite lattice $\mathfrak{b}$, $E=V_{\infty}$, and where the numbers $s_{i}$ take prescribed values. Using the notations of 2.2 and 2.3 we set $H_{0}=\widetilde{V}, \widetilde{V_{i+1}}=\widetilde{V}_{i} \oplus^{\perp} H_{i+1}$, so that each $H_{i}$ is an infinite dimensional Hilbert space, and $\widetilde{E}=\widetilde{\bigoplus}_{i \in N}^{\perp} H_{i}$. On $F$ let us define another form $\Omega_{\infty}$ by $\Omega_{\infty}(x, y)=\lim _{n \rightarrow \infty} \Omega_{n}(x, y)$. Let $H_{\infty}$ be the Hilbert space which is the completion of $F / r \Omega_{\infty}$, let $H=\widetilde{E} \oplus^{\perp} H_{\infty}$ and let $\Omega$ be the form on $H$. For each $i \in N$ we may choose a subspace $F_{i} \subset H_{i}$ together with an isometry $\varphi_{i}:\left(F, \Omega_{i}-\Omega_{i+1}\right) \rightarrow$ $\left(F_{i}, \Omega\right)$ such that

$$
p_{i}(E) \cap F_{i}=0
$$

holds, where $p_{i}: H \rightarrow H_{i}$ is the orthogonal projection. Furthermore let $F_{\infty}=F / r \Omega_{\infty} \subset H_{\infty}$ and $\varphi_{\infty}: F \rightarrow F_{\infty}$ the canonical map. We define

$$
\begin{aligned}
& E^{\prime}:=E \oplus\left\{\sum_{i \in N} \varphi_{i}(x)+\varphi_{\infty}(x) \mid x \in F\right\} \subset H \\
& \Phi^{\prime}:=\Omega \mid E^{\prime} \times E^{\prime} \\
& V^{\prime}:=V .
\end{aligned}
$$

Notice that $\varphi(x)=\sum_{i \in N} \varphi_{i}(x)+\varphi_{\infty}(x) \in H$ exists and has $\Omega(\varphi(x), \varphi(x))=$ $\Omega_{0}(x, x)$. More generally we shall have

$$
\Omega_{i}(x, x)=\Psi_{i}^{\prime}(\varphi(x), \varphi(x)) \quad(x \in F)
$$

if we can show that for $i \in N$ the following equations hold:

$$
V_{i}^{\prime}=V_{i},\left.\Psi_{i}^{\prime}\right|_{E \times E}=\Psi_{i}, r \Psi_{i}^{\prime}=r \Psi_{i}, V_{i-1}^{\perp^{\prime} i-1}=V_{i-1}^{\perp i-1}
$$

(the last one if $i \geqq 1$ ). This, of course, will finish the proof of the proposition for, by (15) the values of the $s_{i}$ are not changed if we pass from $\sqrt{5}$ to $\mathbb{C}^{\prime}=\left(E^{\prime}, \Phi^{\prime}, V^{\prime}\right)$, and by (14) the map $\phi$ is an isomorphism between $\left(F,\left(\Omega_{i}\right)\right)$ and the top of $\mathbb{E}^{\prime}$.

The proof of (15) goes by induction. For $i=0$ we may cite (13). Assume then (15) for $i \leqq n$. Since $V_{n}^{\prime}=V_{n}$ we have $\left.\Psi_{n+1}^{\prime}\right|_{E \times E}=$ $\Psi_{n+1}$ and furthermore $r \Psi_{n+1}^{\prime}=\widetilde{V}_{n} \cap E^{\prime}=\widetilde{V}_{n} \cap(E+\varphi(F))=\widetilde{V}_{n} \cap E=$ $r \Psi_{n+1}\left(\varphi(F) \cap\left(E+\widetilde{V}_{n}\right)=0\right)$. The first equation in (15) is a consequence of the last two, by definition. As to the last equation, notice that $V_{n}^{\perp}=G_{n} \cap E$ and similarly $V_{n}^{\prime \perp^{\prime} n}=G_{n} \cap E^{\prime}$, where $H=H_{n} \oplus^{\perp} G_{n}$. Let $z=y+\varphi(x) \in G_{n} \cap E^{\prime}(y \in E, x \in F)$. We have $0=p_{n}(z)=p_{n}(y)+$ $p_{n}(\varphi(x))$. But $p_{n}(\varphi(x))=\varphi_{n}(x)$, so $x=0$ by (12), showing that $z \epsilon$ $G_{n} \cap E$. This concludes the proof of the proposition. 


\section{Orthogonal decompositions.}

5.1. In a direct orthogonal sum of a countable family of embeddings $c_{i}$ the cardinals $d_{i}, s_{\imath}$ obviously behave additively. Hence it is a matter of course to derive information about the orthogonal decompositions of a given embedding (5 from Theorem 3.1 and the results of Chapter 4. For instance, we have

Proposition. An embedding 5 with nonzero top admits a decomposition $\mathfrak{E}=\mathfrak{C}^{\prime} \bigoplus^{\perp} \mathfrak{C}^{\prime \prime}$ such that $\mathbb{C}^{\prime}$ has zero top and in $\mathfrak{C}^{\prime \prime}$ all $s_{i}^{\prime \prime}$ are zero.

That is, in the summand ${ }^{\prime \prime}$ the cardinal invariants have their trivial values $\left(d_{i}^{\prime \prime}=\boldsymbol{\gamma}_{0}, s_{i}^{\prime \prime}=0\right.$ for all $\left.i\right)$; it is determined completely (up to congruence) by its (nonzero) top, which is the same as that of $\left(5\right.$. If $s_{i} \neq 0$ for infinitely many $i$ then the summand $(5$ ' is uniquely determined, too (4.1, Corollary 1). Otherwise there is a "minimal" choice for ' $\mathcal{C}^{\prime}$ : If $n$ is the largest number with $s_{i} \neq 0$ set $d_{i}^{\prime}=\aleph_{0}$ for $i \leqq n, d_{i}^{\prime}=0$ for $i>n, s_{i}^{\prime}=s_{i}$ for all $i \in N$.

5.2. Neglecting the summand $\mathbb{C}^{\prime \prime}$ in 5.1 (compare the remarks made in 2.6) we are left with the problem of describing the orthogonal decompositions of embeddings with zero top. Such an embedding is characterized by its cardinal invariants $d_{i}, s_{i}$; we say that $\mathfrak{C}$ is of type $\left(\begin{array}{l}d_{0} d_{1} d_{2} \cdots \\ s_{0} s_{1} s_{2} \cdots\end{array}\right)$.

By 3.1 and 4.1 we have

Proposition. Any embedding with zero top can be decomposed into a direct orthogonal sum of embeddings of the following types

$$
\begin{aligned}
& \left(a_{i}\right)\left(\begin{array}{ccccccccc}
\boldsymbol{\aleph}_{0} & \boldsymbol{\aleph}_{0} & \cdots & \boldsymbol{\aleph}_{0} & 0 & 1 & 0 & 0 & \cdots \\
0 & 0 & \cdots & 0 & 0 & 0 & 0 & 0 & \cdots
\end{array}\right) \\
& \left(b_{i}\right)\left(\begin{array}{cccccccc}
\boldsymbol{\aleph}_{0} & \boldsymbol{\aleph}_{0} & \cdots & \boldsymbol{\aleph}_{0} & \boldsymbol{\aleph}_{0} & 0 & 0 & \cdots \\
0 & 0 & \cdots & 0 & 1 & 0 & 0 & \cdots
\end{array}\right)
\end{aligned}
$$

(in both case the 1 stands in the ith column, $i \geqq 0$ ).

The number of copies of type $\left(b_{i}\right)$ equals $s_{i}$ and is therefore uniquely determined. If the lattice $\mathfrak{b}$ is finite the decomposition can be made unique by dropping "superfluous" summands of type $\left(a_{2}\right)$ :

Let $m(n)$ be the largest number for which $d_{m} \neq 0\left(s_{n} \neq 0\right)$. If $m \leqq n$ no summands of type $\left(a_{i}\right)$ are needed. If $m=n+1$ it suffices to take $d_{m}$ copies of type $\left(a_{m}\right)$ whereas, if $m \geqq n+2$, we admit $d_{m}$ copies of type $\left(a_{m}\right)$ and $d_{m-1}$ copies of type $\left(a_{m-1}\right)$. 
5.3. Let us consider some simple cases of special interest.

ExAmple 1. An embedding $\mathbb{E}$ is $\perp$-dense if and only if it is of type $\left(\begin{array}{cccccc}d_{0} & 0 & d_{2} & 0 & 0 & \cdots \\ s_{0} & 0 & 0 & 0 & 0 & \cdots\end{array}\right)$. If $s_{0}+d_{2} \neq 0$, $\sqrt{5}$ is an orthogonal sum of $s_{0}$ copies $\left(b_{0}\right)$ and $d_{2}$ copies $\left(a_{2}\right)$. If $s_{0}=0=d_{2}$ we have $V=E$, a sum of $d_{0}$ copies $\left(a_{0}\right)$.

ExAMPLE 2. An embedding $\sqrt{ }$ is $\perp$-closed if and only if it is of type $\left(\begin{array}{ccccccc}d_{0} & d_{1} & 0 & d_{3} & 0 & 0 & \cdots \\ 0 & s_{1} & 0 & 0 & 0 & 0 & \cdots\end{array}\right)$. If $s_{1}+d_{3} \neq 0$, $\sqrt{5}$ is an orthogonal sum of $s_{1}$ copies $\left(b_{1}\right)$ and $d_{3}$ copies $\left(a_{3}\right)$. If $s_{1}=0=d_{3}$ we have $V+V^{\perp}=E$, a sum of $d_{0}$ copies $\left(a_{0}\right)$ and $d_{1}$ copies $\left(a_{1}\right)$.

Example 1 has already been settled in [5], [8], but Example 2 only in the case $\operatorname{dim} E / V+V^{\perp}<\infty$. Combining the two we get: $\sqrt{5}$ is the orthogonal sum of a $\perp$-dense and a $\perp$-closed embedding if and only if $E=V_{2}+V_{2}^{\perp_{2}}$, if and only if it is of type $\left(\begin{array}{ccccccc}d_{0} & d_{1} & d_{2} & d_{3} & 0 & 0 & \cdots \\ s_{0} & s_{1} & 0 & 0 & 0 & 0 & \cdots\end{array}\right)$.

To round off the discussion of such embeddings we shall give concrete representatives for $\left(b_{0}\right),\left(a_{2}\right),\left(b_{1}\right),\left(a_{3}\right)\left(\left(a_{0}\right)\right.$ and $\left(a_{1}\right)$ being trivial).

$\left(b_{0}\right): \quad E=V \oplus \boldsymbol{R} f, V$ with orthonormal basis $\left(v_{i}\right)_{i \in N}, \Phi(f, f)=1$, $\Phi\left(f, v_{\imath}\right)=2^{-(\imath+1) / 2}(i \geqq 0)$.

$\left(a_{2}\right)$ : same as $\left(b_{0}\right)$, but $\Phi\left(f, v_{i}\right)=2^{-(i+2) / 2}$.

$\left(b_{1}\right): \quad E=\left(V \oplus^{\perp} V^{\perp}\right) \oplus \boldsymbol{R} f, V$ and $V^{\perp}$ with orthonormal bases $\left(v_{2 j}\right)_{j \in N}$ and $\left(v_{2 j+1}\right)_{j \in N}$ respectively, $\Phi(f, f)=1, \Phi\left(f, v_{i}\right)=2^{-(i+1) / 2}$.

$\left(a_{3}\right)$ : same as $\left(b_{1}\right)$, but $\Phi\left(f, v_{i}\right)=2^{-(\imath+2) / 2}$.

The verifications are left to the reader (the crucial thing is that in $\left(b_{0}\right),\left(b_{1}\right)$ we have $\sum_{i \in N} \Phi\left(f, v_{i}\right)^{2}=1$, whilst in $\left(a_{2}\right),\left(a_{3}\right)$ we must have $\left.\sum_{\imath \in N} \Phi\left(f, v_{i}\right)^{2}<1\right)$.

\section{REFERENCES}

1. P. Gabriel, Représentations Indécomposables, Séminaire Bourbaki, vol. 1973/74, exposé $\mathrm{n}^{\circ}$ 444, Springer Lecture Notes 431.

2. H. Gross, On Witt's theorem in the denumerably infinite case, II, Math. Ann., 170 (1967), 145-165.

3. - Eine Bemerkung zu dichten Unterräumen reeller quadratischer Räume, Comment. Math. Helv., 45 (1970), 472-493.

4. - Isomorphisms between lattices of linear subspaces which are induced by isometrics, J. Algebra, 49 (1977), 537-546.

5. — Quadratic forms in infinite dimensional spaces, Progress in Mathematics Vol. 1, Birkhauser Basel-Boston, 1979.

6. I. Kaplansky, Forms in infinite dimensional spaces, An. Acad. Brasil. Cienc., 22 (1950), 1-17.

7. H. G. Quebbemann, R. Scharlau, W. Scharlau and M. Schulte, Quadratische Formen in additiven Kategorien, Bull. Soc. Math. France, Mémoire 48 (1976), 93-101.

8. U. Schneider, Beiträge zur Theorie der sesquilinearen Räume unendlicher Di- 
mension, Ph. D. thesis, University of Zürich, 1975.

Received September 18, 1978. This paper was written during the author's stay at the Forschungsinstitut für Mathematik, ETH Zürich.

Forschungsinstitut Für Mathematik

ETH

ZURICH, SwitzerLaND 


\section{PACIFIC JOURNAL OF MATHEMATICS}

\section{EDITORS}

DoNALD BABBITT (Managing Editor)

University of California

Los Angeles, California 90024

HUGo Rossi

University of Utah

Salt Lake City, UT 84112

C. C. MOORE and ANDREW OGG

University of California

Berkeley, CA 94720
J. DUGUNDJI

Department of Mathematics University of Southern Californı Los Angeles, California 90007

R. Finn and J. Milgram Stanford University Stanford, California 94305

\section{ASSOCIATE EDITORS}

E. F. BECKENBACH

B. H. Neumann
F. WOLF

K. YOSHIDA

\section{SUPPORTING INSTITUTIONS}

UNIVERSITY OF BRITISH COLUMBIA CALIFORNIA INSTITUTE OF TECHNOLOGY UNIVERSITY OF CALIFORNIA MONTANA STATE UNIVERSITY UNIVERSITY OF NEVADA, RENO NEW MEXICO STATE UNIVERSITY OREGON STATE UNIVERSITY UNIVERSITY OF OREGON
UNIVERSITY OF SOUTHERN CALIFORNIA STANFORD UNIVERSITY UNIVERSITY OF HAWAII UNIVERSITY OF TOKYO UNIVERSITY OF UTAH WASHINGTON STATE UNIVERSITY UNIVERSITY OF WASHINGTON 


\section{Pacific Journal of Mathematics}

Vol. 82, No. $1 \quad$ January, 1979

Werner Bäni, Subspaces of positive definite inner product spaces of countable dimension ...................................... 1

Marilyn Breen, The dimension of the kernel of a planar set..............

Kenneth Alfred Byrd, Right self-injective rings whose essential right ideals

are two-sided

Patrick Cousot and Radhia Cousot, Constructive versions of Tarski's fixed

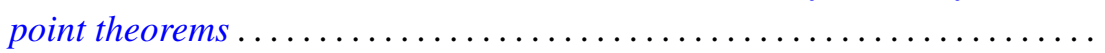

Ralph S. Freese, William A. Lampe and Walter Fuller Taylor, Congruence lattices of algebras of fixed similarity type. $I \ldots \ldots \ldots \ldots \ldots \ldots \ldots$

Cameron Gordon and Richard A. Litherland, On a theorem of Murasugi .....

Mauricio A. Gutiérrez, Concordance and homotopy. I. Fundamental

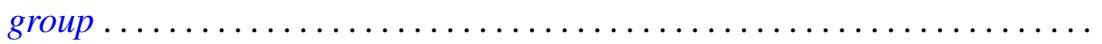

Richard I. Hartley, Metabelian representations of knot groups .............

Ted Hurley, Intersections of terms of polycentral series of free groups and free

Lie algebras ........................................

Roy Andrew Johnson, Some relationships between measures ............ 117

Oldřich Kowalski, On unitary automorphisms of solvable Lie algebras .......

Kee Yuen Lam, $K O$-equivalences and existence of nonsingular bilinear

maps...................................................

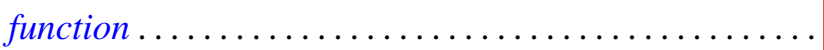

Robert A. Messer and Alden H. Wright, Embedding open 3-manifolds in compact 3-manifolds ............................

Gerald Ira Myerson, A combinatorial problem in finite fields. I . .

James Nelson, Jr. and Mohan S. Putcha, Word equations in a band of paths.

Baburao Govindrao Pachpatte and S. M. Singare, Discrete generalized Gronwall inequalities in three independent variables . .

William Lindall Paschke and Norberto Salinas, $C^{*}$-algebras associated with free products of groups ........................

Bruce Reznick, Banach spaces with polynomial norms ....

David Rusin, What is the probability that two elements of a finite group

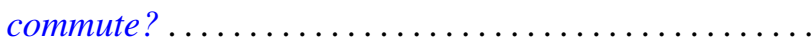

M. Shafii-Mousavi and Zbigniew Zielezny, On hypoelliptic differential operators of constant strength ...

Joseph Gail Stampfli, On selfadjoint derivation ranges .... . . .

Robert Charles Thompson, The case of equality in the matrix-valued triangle

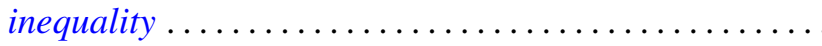

Marie Angela Vitulli, The obstruction of the formal moduli space in the negatively graded case. 